\title{
Electromagnetic excitation of nuclei and neutron evaporation in ultrarelativistic ultraperipheral heavy ion collisions
}

\author{
Mariola Kłusek-Gawenda,, , * Michał Ciemała,, , \\ Wolfgang Schäfer, ${ }^{1, f}$ and Antoni Szczurek ${ }^{1,2}$,\$ \\ ${ }^{1}$ Institute of Nuclear Physics PAN, PL-31-342 Cracow, Poland \\ 2 University of Rzeszów, PL-35-959 Rzeszów, Poland
}

(Dated: August 16, 2021)

\begin{abstract}
We present a new approach for calculating electromagnetic excitation of nuclei as well as probabilities of emission and distributions of neutrons from decays of excited nuclear systems for ultrarelativistic, ultraperipheral heavy ion collisions. Excitation functions for $\gamma+\mathrm{Pb} \rightarrow \mathrm{Pb}^{*}$ are parametrized using physics-motivated components: excitation of giant resonances, quasi-deuteron absorption mechanism, excitation of nucleon resonances as well as high-energy dissociation of protons and neutrons. Neutron emission (up to 10 neutrons) from low-energy excitations of ${ }^{208} \mathrm{~Pb}$ is modelled in terms of the Hauser-Feshbach formalism. The probabilities of a given number of neutrons are calculated as a function of excitation energy in a Monte-Carlo code GEMINI. These functions are parametrized by smooth analytical functions. The results are compared to appropriate data for the $\gamma \mathrm{Pb} \rightarrow \mathrm{Pb}^{*} \rightarrow \mathrm{kn}$ reaction. As an example the approach is used for calculating electromagnetic excitation in UPC processes. Both single photon and double photon excitations are included and discussed. Topological cross sections with a given number of neutrons in forward and backward directions are calculated in the calculation at the LHC energies. Excitation functions are presented. The results of calculation are compared with SPS and more recent ALICE experimental data and good agreement is achieved.
\end{abstract}

PACS numbers: 25.75.-q (Heavy-ion nuclear reactions relativistic)

25.20.-x (photonuclear reactions).

*Electronic address: mariola.klusek@ifj.edu.pl

${ }^{\dagger}$ Electronic address: michal.ciemala@ifj.edu.pl

${ }_{\ddagger}^{\ddagger}$ Electronic address: wolfgang.schafer@ifj.edu.pl

§Electronic address: antoni.szczurek@ifj.edu.pl 


\section{INTRODUCTION}

The exclusive production of mesons, pairs of quark and antiquark, pairs of leptons or other Standard Model particles in ultraperipheral heavy ion collisions (UPC) has been attracting much attention [1, 2]. A measurement of such reactions at the high energies of present day colliders (RHIC, LHC) often requires special triggers. Large charges of colliding ions lead to the production of huge fluxes of associated photons. These photons when scattered on the collision partner lead to its excitation. As will be discussed in the present paper lowenergy excitations $\left(E^{*}<50 \mathrm{MeV}\right)$ play especially important role. The low-energy excited nuclear heavy systems, close to giant resonance region, decay predominantly via emission of neutrons. Because the energy of the neutrons in the nucleus rest frame is rather small $(\sim 10$ - $20 \mathrm{MeV}$ ), in UPCs the neutrons are emitted in very small cones around beam directions. Such neutrons can be registered by the so-called zero-degree calorimeters (ZDC's) which are associated with many high-energy detectors, such as e.g. STAR at RHIC [3] and ALICE at LHC [4].

In the present paper we wish to present our approach which includes description of photoexcitation of nuclei and decay of excited nuclei in the framework of Hauser-Feshbach theory. Results of our calculations for $\gamma \mathrm{Pb} \rightarrow \mathrm{Pb}^{*} \mathrm{k} \mathrm{n}$ are confronted with existing experimental data. Then topological cross sections with a given number of neutrons in ion-ion collisions are calculated and compared to RHIC and LHC data. We discuss the role of single and double-photon excitations. We present simple parametrizations of the relevant impact parameter profiles which can be conveniently used for a multitude of central final states produced in diffractive or $\gamma \gamma$ subprocesses, such as vector mesons, leptons, pions, etc.

\section{FORMALISM}

\section{A. Electromagnetic excitation in UPC}

In this subsection we collect the classical probability calculus methods needed [5-7] to describe the electromagnetic excitation of ions in UPCs due to multiple photon exchanges.

From the usual Weizsäcker-Williams flux of photons $N_{A}(E, \mathbf{b})$ and the total photoabsorption cross section $\sigma_{\text {tot }}(\gamma A ; E)$ discussed in section III, we introduce the mean number of photons absorbed by a nucleus $A_{2}$ in the collision with nucleus $A_{1}$ :

$$
\bar{n}_{A_{2}}(\mathbf{b}) \equiv \int_{E_{\min }}^{\infty} d E N_{A_{1}}(E, \mathbf{b}) \sigma_{\mathrm{tot}}\left(\gamma A_{2} ; E\right) .
$$

Here the upper limit in the integral is only formal: the photon flux (see any of the reviews [1])

$$
N_{A}(E, \mathbf{b})=\frac{1}{E} \frac{Z^{2} \alpha_{\mathrm{em}}}{\pi^{2}} \frac{1}{\mathbf{b}^{2}} \xi^{2} K_{1}^{2}(\xi), \xi=\frac{E b}{\gamma_{\mathrm{lab}}}
$$

implicitly contains a cut off in energy. Above $E$ is the photon energy in the rest frame of nucleus $A_{2}, Z$ is the nuclear charge and $K_{1}$ is a modified Bessel function. The boost to the rest frame of nucleus $A_{2}$ is given by

$$
\gamma_{\mathrm{lab}}=2 \gamma_{\mathrm{cm}}^{2}-1, \gamma_{\mathrm{cm}}=\frac{\sqrt{s_{N N}}}{2 m_{N}}
$$


The lower limit of integration $E_{\text {min }}$ is the threshold for photoexcitation. As in practice $\bar{n}_{A}(\mathbf{b}) \ll 1$, for statistically independent absorption, we can state the probability of absorption of $n$ photons at impact parameter $\mathbf{b}$ in the Poissonian form

$$
w_{n}(\mathbf{b})=\frac{\left(\bar{n}_{A}(\mathbf{b})\right)^{n}}{n !} \exp \left[-\bar{n}_{A}(\mathbf{b})\right] .
$$

We define the probability density for a single photon to excite nucleus $A_{2}$ in a collision at impact parameter $\mathbf{b}$ of the $A_{1}-A_{2}$ collision as

$$
p_{A_{2}}^{(1)}(E, \mathbf{b})=\frac{N_{A_{1}}(E, \mathbf{b}) \sigma_{\text {tot }}\left(\gamma A_{2} ; E\right)}{\bar{n}_{A_{2}}(\mathbf{b})},
$$

which is fulfilled, at each $\mathbf{b}$

$$
\int_{E_{\min }}^{\infty} d E p_{A_{2}}^{(1)}(E, \mathbf{b})=1
$$

Still under the assumption of statistical independence, $n$ photons will excite the nucleus with the probability density

$$
p_{A_{2}}^{(n)}(E, \mathbf{b})=\int d E_{1} d E_{2} \ldots d E_{n} \delta\left(E-\sum_{j=1}^{n} E_{j}\right) p_{A_{2}}^{(1)}\left(E_{1}, \mathbf{b}\right) p_{A_{2}}^{(1)}\left(E_{2}, \mathbf{b}\right) \ldots p_{A_{2}}^{(1)}\left(E_{n}, \mathbf{b}\right)
$$

All the $n$-photon probability densities are properly normalized:

$$
\int_{E_{\min }}^{\infty} d E p_{A_{2}}^{(n)}(E, \mathbf{b})=1
$$

Below we will explicitly calculate processes up to $n=2$ photon exchanges, see diagrams in Figs. 1, 2,

Then, the probability for excitation of nucleus $A_{2}$ in the $n$-photon process is given by

$$
w_{n}(\mathbf{b}) p_{A_{2}}^{(n)}(E, \mathbf{b}) \text {. }
$$

We should sum over all numbers of photons

$$
\frac{d P_{A_{2}}^{\mathrm{exc}}(\mathbf{b})}{d E}=\sum_{n} w_{n}(\mathbf{b}) p_{A_{2}}^{(n)}(E, \mathbf{b}) \approx \exp \left[-\bar{n}_{A_{2}}(\mathbf{b})\right] N_{A_{1}}(E, \mathbf{b}) \sigma_{\mathrm{tot}}\left(\gamma A_{2} ; E\right),
$$

where we indicated that we expect the single-photon absorption to dominate. Notice that this may in practice depend on impact parameter. The total probability for the nucleus to be excited is then

$$
P_{A_{2}}^{\operatorname{exc}}(\mathbf{b})=\int d E \frac{d P_{A_{2}}^{\operatorname{exc}}(\mathbf{b})}{d E}=1-\exp \left[-\bar{n}_{A_{2}}(\mathbf{b})\right] \approx \bar{n}_{A_{2}}(\mathbf{b}) \exp \left[-\bar{n}_{A_{2}}(\mathbf{b})\right] .
$$

The excitation cross section is then

$$
\sigma_{\text {tot }}\left(A_{1} A_{2} \rightarrow A_{1} A_{2}^{*}\right)=\int d^{2} \mathbf{b} P_{\text {surv }}(\mathbf{b}) P_{A_{2}}^{\text {exc }}(\mathbf{b})=\int d^{2} \mathbf{b} P_{\text {surv }}(\mathbf{b})\left(1-\exp \left[-\bar{n}_{A_{2}}(\mathbf{b})\right]\right) .
$$


Sometimes we are interested in the excitation cross section containing only excitations up to $E_{\max } \lesssim 100 \mathrm{MeV}$, then we can calculate the cross section from

$$
\begin{aligned}
& \sigma_{\text {tot }}\left(A_{1} A_{2} \rightarrow A_{1} A_{2}^{*} ; E_{\max }\right) \approx \int d^{2} \mathbf{b} P_{\text {surv }}(\mathbf{b}) \exp \left[-\bar{n}_{A_{2}}(\mathbf{b})\right] \\
& \times \int_{E_{\min }}^{E_{\max }} d E N_{A_{1}}(E, \mathbf{b}) \sigma_{\mathrm{tot}}\left(\gamma A_{2} ; E\right)
\end{aligned}
$$

Here

$$
P_{\text {surv }}(\mathbf{b}) \sim \theta\left(|\mathbf{b}|-\left(R_{A_{1}}+R_{A_{2}}\right)\right)
$$

is the probability for the nuclei to survive the collision without additional strong interactions. As is apparent

$$
w_{0}(\mathbf{b})=\exp \left[-\bar{n}_{A_{2}}(\mathbf{b})\right]
$$

is the contribution to the survival probability from the electromagnetic dissociation channels. The cross section for mutual electromagnetic dissociation is simply obtained from

$$
\sigma_{\mathrm{tot}}\left(A_{1} A_{2} \rightarrow A_{1}^{*} A_{2}^{*}\right)=\int d^{2} \mathbf{b} P_{\mathrm{surv}}(\mathbf{b}) P_{A_{2}}^{\mathrm{exc}}(\mathbf{b}) P_{A_{1}}^{\mathrm{exc}}(\mathbf{b})
$$

In Fig. 2 we show the situation when two photons emitted by one of the colliding nuclei

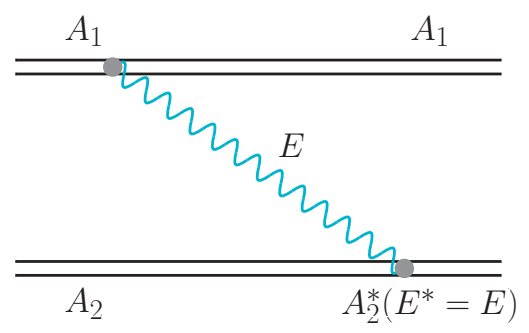

FIG. 1: Single excitation in UPCs.

hit the second nucleus. Finally in Fig. 3 we show as an example the case when each of

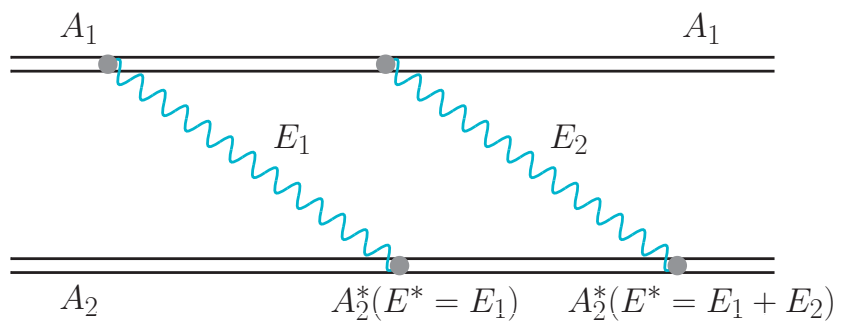

FIG. 2: Double excitation in UPCs.

the nuclei emit a photon which excites then the collision partner. We shall call this case mutual excitations. The diagram shows a minimal mechanism needed to excite both nuclei simultaneously. Higher-order diagrams are possible too. 


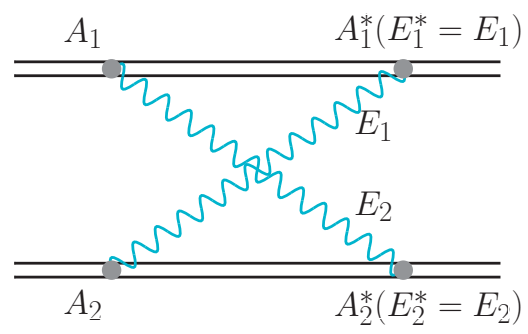

FIG. 3: Minimal mechanism for mutual excitation in UPCs.

\section{B. Dissociation into specific final states}

In the present paper we are interested mainly in final states that contain a few neutrons, and want to study excitation cross sections as a function of neutron multiplicity (a type of "topological cross sections"). Here the crucial input are the fractions $f(E, k)$ of a final state with $k$ neutrons coming from the decay of an excited nucleus at excitation energy $E$. With their help, we can calculate the impact parameter profiles for processes with $k$ evaporated neutrons as:

$$
\frac{d P_{A_{2}}^{\mathrm{exc}}(\mathbf{b}, k)}{d E}=f(E, k) \cdot \sum_{n} w_{n}(\mathbf{b}) p_{A_{2}}^{(n)}(E, \mathbf{b}) \approx f(E, k) p_{A_{2}}^{(1)}(E, \mathbf{b}) \bar{n}_{A_{2}}(\mathbf{b}) \exp \left[-\bar{n}_{A_{2}}(\mathbf{b})\right],
$$

and, correspondingly:

$$
P_{A_{2}}^{\mathrm{exc}}(\mathbf{b}, k)=\int_{E_{\min }}^{E_{\max }} d E \frac{d P_{A_{2}}^{\mathrm{exc}}(\mathbf{b})}{d E} .
$$

In Fig. 4 we plot these distributions as a function of impact parameter for $k=1,2,3$ at $\gamma_{\mathrm{cm}}=1470$. The cross section for $k$-neutron excitation is then

$$
\sigma\left(A_{1} A_{2} \rightarrow A_{1}(k \mathrm{~N}, X)\right)=\int d^{2} \mathbf{b} P_{\text {surv }}(\mathbf{b}) P_{A_{2}}^{\operatorname{exc}}(\mathbf{b}, k) .
$$

Of course we are confined to low-neutron multiplicities, as final states of large number of neutron $(k)$ can be produced by processes in the energy region $E>E_{\max }$ which we do not model so far. Analogously, the mutual excitation cross sections with $m$ and $k$ neutrons in the debris of nucleus $A_{1}, A_{2}$, respectively, is

$$
\sigma\left(A_{1} A_{2} \rightarrow(m \mathrm{~N}, X)(k \mathrm{~N}, Y)\right)=\int d^{2} \mathbf{b} P_{\text {surv }}(\mathbf{b}) P_{A_{1}}^{\mathrm{exc}}(\mathbf{b}, m) P_{A_{2}}^{\mathrm{exc}}(\mathbf{b}, k) .
$$




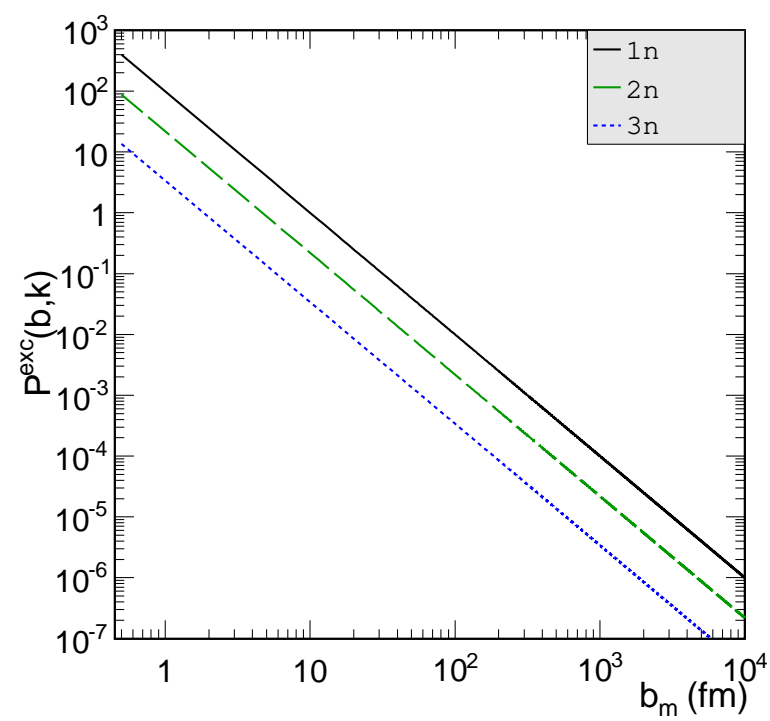

FIG. 4: Impact parameter profile for processes with evaporation of $k$ neutrons for $k=1,2,3$.

\section{PHOTON-INDUCED EXCITATION OF NUCLEI AND NEUTRON EVAPO- RATION}

To evaluate the photoabsorption probabilities, we need a parametrization of the total phtotabsorption cross section over a broad range of energies. Here we are not interested in a microscopic modelling of the different mechanisms play an important role at different energies, but rather in a fit of empirical data.

At the lowest energies of relevance, photoabsorption is dominated by giant resonances.

The energy dependence of the cross section for the giant dipole resonance (GDR) component $\left(\sigma_{\mathrm{GDR}}\right)$ is parametrized following Ref. [8, 9]:

$$
\sigma_{\mathrm{GDR}}=\frac{2}{\pi} \sigma_{T R K} \frac{E^{2} \Gamma_{r}}{\left(E^{2}-E_{r}^{2}\right)^{2}+\left(E \Gamma_{r}\right)^{2}} S_{r} .
$$

The parameters

$$
\sigma_{T R K}=60 \frac{N Z}{A} \mathrm{mb} \mathrm{MeV}, E_{r}=13.373 \mathrm{MeV}, \Gamma_{r}=3.938 \mathrm{MeV}, S_{r}=1.33716 .
$$

are taken from Ref. [10].

At somewhat larger energies a so-called quasi-deuteron contribution plays important role and following [11] is parametrized as

$$
\sigma_{\mathrm{QD}}=6.5 \frac{N Z}{A} \sigma_{d} f(E)
$$

where

$$
\sigma_{d}=61.2 \frac{(E-2.224)^{3 / 2}}{E^{3}} \mathrm{mb}
$$




$$
\begin{aligned}
f(E<20 M e V) & =\exp (-73.3 / E) \\
f(20<E<140 M e V) & =8.3714 \times 10^{-2} \\
& -9.8343 \times 10^{-3} E+4.1222 \times 10^{-4} E^{2} \\
& -3.4762 \times 10^{-6} E^{3}+9.3537 \times 10^{-9} E^{4} \\
f(E>140 M e V) & =\exp (-24.2 / E) .
\end{aligned}
$$

Above a photon energy $E_{\gamma}>100 \mathrm{MeV}$ the nucleon resonances are taken into account. The $\Delta$ resonance being the dominant feature of the excitation spectrum. We parametrize this region of the photoabsorption cross section with the help of a Gaussian function

$$
\sigma_{\text {Gauss }}=\frac{C_{G}}{\sigma_{G} \sqrt{2 \pi}} \exp \left(\frac{-\left(E-\mu_{G}\right)^{2}}{2 \sigma_{G}^{2}}\right),
$$

where $C_{G}=23$ barn $\mathrm{MeV}, \sigma_{G}=110 \mathrm{MeV}, \mu_{G}=350 \mathrm{MeV}$.

Above $E_{\gamma}>0.5 \mathrm{GeV}$ the resonant contributions disappear and the continuum related to break-up of nucleons starts to be important. The corresponding total cross section (forward amplitude of photon elastic scattering) is usually parametrized by exchange of pomeron at very large energies and subleading reggeons at intermediate energies. In our simple parametrization the pomeron exchange contribution is parametrized as a constant and slightly phenomenological function is used to represent the reggeon exchange contribution.

For the highest energy part $\left(E_{\gamma}>8 \mathrm{GeV}\right)$ we use a simple form given in [12] (below $\left.\omega_{0}=80 \mathrm{GeV}\right)$

$$
\sigma_{\gamma A}=\left(15.2+0.06 \ln ^{2}\left(\frac{E}{\omega_{0}}\right)\right) \mathrm{mb}
$$

This multicomponent parametrization is compared to the experimental data for photoabsorption on lead [13] in Fig. 5. The quality of the description of the data is fully sufficient for our purpose.

\section{A. Decays of excited nuclear systems}

The calculation of probability of evaporated neutron multiplicity as a function of ${ }^{208} \mathrm{~Pb}$ excitation energy was performed with the help of a Monte Carlo code GEMINI++ [14]. In this code the evaporation process is described by Hauser-Feshbach formalism [15], in which the decay width for evaporation of a particle $i$ from the compound nucleus with excitation energy $E^{*}$ and spin $S_{C N}$ is:

$$
\Gamma_{i}=\frac{1}{2 \pi \rho\left(E^{*}, S_{C N}\right)} \int d \epsilon \sum_{S_{d}=0}^{\infty} \sum_{J=\left|S_{C N}-S_{d}\right|}^{S_{C N}-S_{d}} \sum_{\ell=\left|J-S_{i}\right|}^{J+S_{i}} T_{\ell}(\epsilon) \rho\left(E^{*}-B_{i}-\epsilon, S_{d}\right),
$$

where $S_{d}$ is the spin of the daughter nucleus, $S_{i}, J$ and $\ell$ are spin, total and angular momentum of the evaporated particle, $\epsilon, B_{i}$ are kinetic and separation energies, $T_{\ell}$ is its transmission coefficient, $\rho$ and $\rho_{C N}$ are level densities of the daughter and compound nucleus, which can be calculated from the formula:

$$
\rho\left(E^{*}, S\right) \propto(2 S+1) \exp (2 \sqrt{a(U, S) U}),
$$




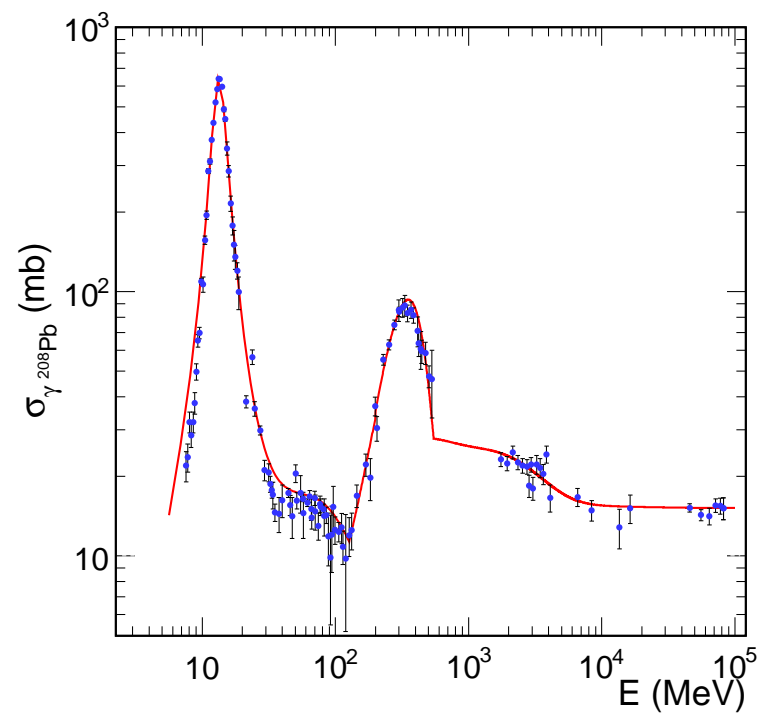

FIG. 5: Photoabsorption cross section for the $\gamma^{208} \mathrm{~Pb} \rightarrow{ }^{208} \mathrm{~Pb}$ reaction

where $U=E^{*}-E_{\text {rot }}(S)-\delta P$ is thermal excitation energy calculated by taking into account pairing corrections to the empirical mass formula $(\delta P)$ and rotational energy $E_{\text {rot }}(S)$. In calculations the separation energies $B_{i}$, nuclear masses, shell and pairing corrections were used according to Ref. [16]. Level density parameter $a(U, S)$ was calculated as:

$$
a(U, S)=\tilde{a}(U)\left(1-h\left(U / \eta+S / S_{\eta}\right) \frac{\delta W}{U}\right)
$$

where $\delta W$ is the shell correction to the liquid-drop mass and $\tilde{a}$ is smoothed level-density parameter, the function specifying the rate of fadeout is $h(x)=\tanh x$, the fadeout parameter $\eta$ was equal $18.52 \mathrm{MeV}$ and the parameter $S_{\eta}$ was set to $50 \hbar$.

The smoothed level density parametrization depends on the nuclei excitation energy as:

$$
\tilde{a}(U)=\frac{A}{k_{\infty}-\left(k_{\infty}-k_{0}\right) \exp \left(-\frac{\kappa}{k_{\infty}-k_{0}} \frac{U}{A}\right)},
$$

where $k_{0}=7.3, k_{\infty}=12$ and $\kappa=0.00517 \exp (0.0345 A)$ [14].

We assume that excited nucleus is formed with angular momentum equal to 0 (which we believe is a good approximation for photoproduction) and full energy is used for excitation. The calculation is done with energy step of $1 \mathrm{MeV}$. For each excitation energy $10^{6}$ events (decays) were generated. Finally neutron emissions probabilities were obtained from the MC sample for each excitation energy (see histogram in Fig. 6).

The fractions of events with a $k$-neutron final state at excitation energy $E^{*}$ can be well fitted by a sum of the following purely empirical functions:

$$
\begin{gathered}
f\left(E^{*}, k\right)=f^{e x p}\left(E^{*}, k\right)+f^{\text {Gauss }}\left(E^{*}, k\right) . \\
f^{e x p}\left(E^{*}, k\right)=C_{e}\left(E^{*}-\mu_{e}\right)^{2} \exp \left(\frac{-\left(E^{*}-\mu_{e}\right)}{\sigma_{e}}\right),
\end{gathered}
$$




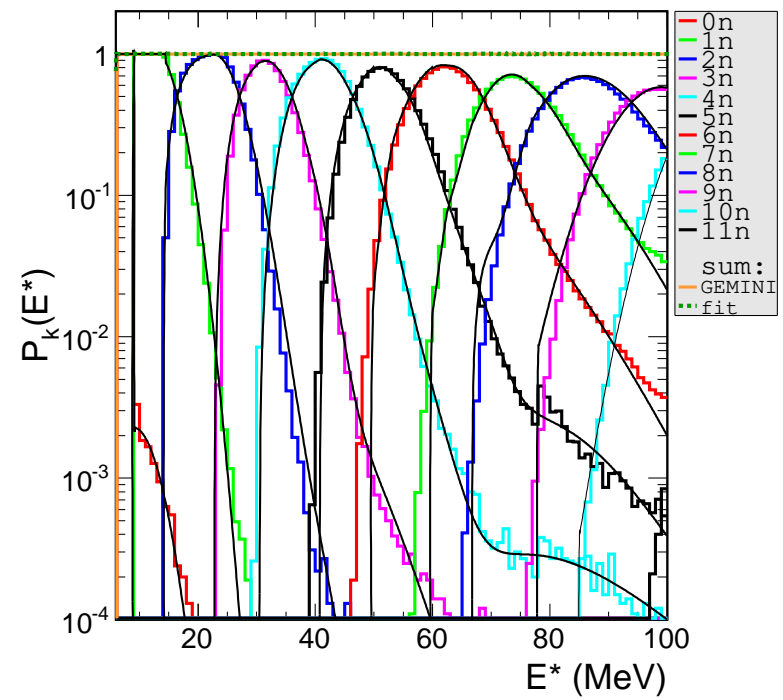

FIG. 6: Probability of neutron multiplicity as a function of excitation energy $\left(E^{*}\right)$ of ${ }^{208} \mathrm{~Pb}$ nuclei.

$$
f^{\text {Gauss }}\left(E^{*}, k\right)=\frac{C_{G}}{\sigma_{G} \sqrt{2 \pi}} \exp \left(\frac{-\left(E^{*}-\mu_{G}\right)^{2}}{2 \sigma_{G}^{2}}\right) .
$$

The parameters of the phenomenological functions found in the fit are collected in Table I] and can be used in any calculations.

To ensure that probabilities always add up to unity, in practice we impose

$$
\begin{aligned}
& f\left(E^{*}, 2\right)=1-f\left(E^{*}, 1\right) \text { for } E^{*}<22 \mathrm{MeV} \\
& f\left(E^{*}, 3\right)=1-f\left(E^{*}, 1\right)-f\left(E^{*}, 2\right) \text { for } E^{*}<30 \mathrm{MeV}
\end{aligned}
$$

and similarly for higher $k$.

\section{B. Excitation functions for the $\gamma \mathbf{P b} \rightarrow \mathbf{P b}^{*} \rightarrow \mathbf{k} \mathbf{n}$ reaction}

Using photoabsorption cross section shown in Fig. [5 and probability to emit a fixed number of neutrons ( $\mathrm{k}$ ) obtained as described in section [II we can calculate photon-induced excitation function with a given number of associated neutrons. The results are shown in Fig. 7, 8, 9,

Quite a good agreement with the world data is obtained. This is quite surprising given that our calculation implicitly assumes equilibration of the nuclear system (Hauser-Feshbach formalism) formed after absorption of the photon. If we assumed that part of the energy of the photon would escape before equilibration of the nuclear system (due to pre-equilibrium processes) the agreement with the data would be much worse. Having proven usefulness of our approach we can proceed to the excitation of nuclei in UPCs and related production of neutrons from the excited nuclear systems. In the following we shall present the results of the formalism discussed above. 
TABLE I: Parameters of fit functions for probability of neutron multiplicity $P_{k}\left(E^{*}\right)$.

\begin{tabular}{|c||c|c|c||c|c|c|}
\hline Number of neutrons & $C_{e}$ & $\mu_{e}$ & $\sigma_{e}$ & $C_{G}$ & $\mu_{G}$ & $\sigma_{G}$ \\
\hline \hline 0 & 0 & - & - & 0.02 & 9 & 3.5 \\
\hline 1 & 0 & - & - & 9.9 & 12 & 3.5 \\
\hline 2 & 0.08 & 21.6 & 1.7 & 10.3 & 21.5 & 4.2 \\
\hline 3 & 0.0015 & 38 & 1.2 & 9.4 & 31.5 & 4.2 \\
& 0.0005 & 40 & 2.6 & & & \\
\hline 4 & 0.0012 & 45 & 2.3 & 11 & 41 & 4.8 \\
& 0.000008 & 62 & 8 & & & \\
\hline 5 & 0.03 & 53 & 2.6 & 8.7 & 51 & 4.3 \\
& 0.00015 & 72 & 4.9 & & & \\
\hline 6 & 0.023 & 62.2 & 2.8 & 11.5 & 61.8 & 5.5 \\
& 0.003 & 68 & 4.35 & & & \\
\hline 7 & 0.015 & 76 & 4 & 9.5 & 73.5 & 5.3 \\
\hline 8 & 0 & - & - & 8.8 & 84 & 5.3 \\
\hline 9 & 0 & - & - & 10.5 & 99 & 7.2 \\
\hline 10 & 0 & - & - & 10.1 & 110 & 6.5 \\
\hline
\end{tabular}

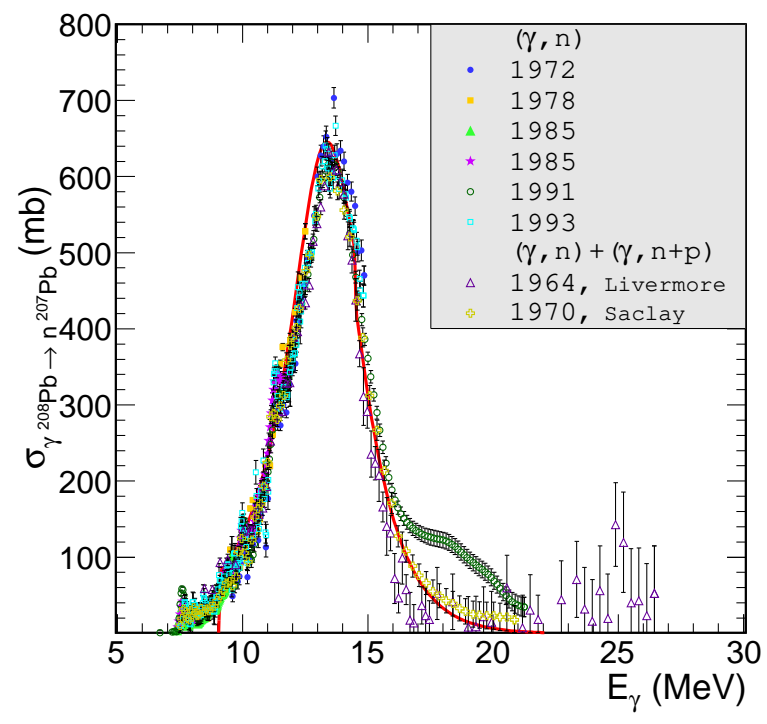

FIG. 7: Excitation energy for the $\gamma^{208} \mathrm{~Pb} \rightarrow \mathrm{n}^{207} \mathrm{~Pb}$ reaction. Experimental data are from Refs. [17 24]. 


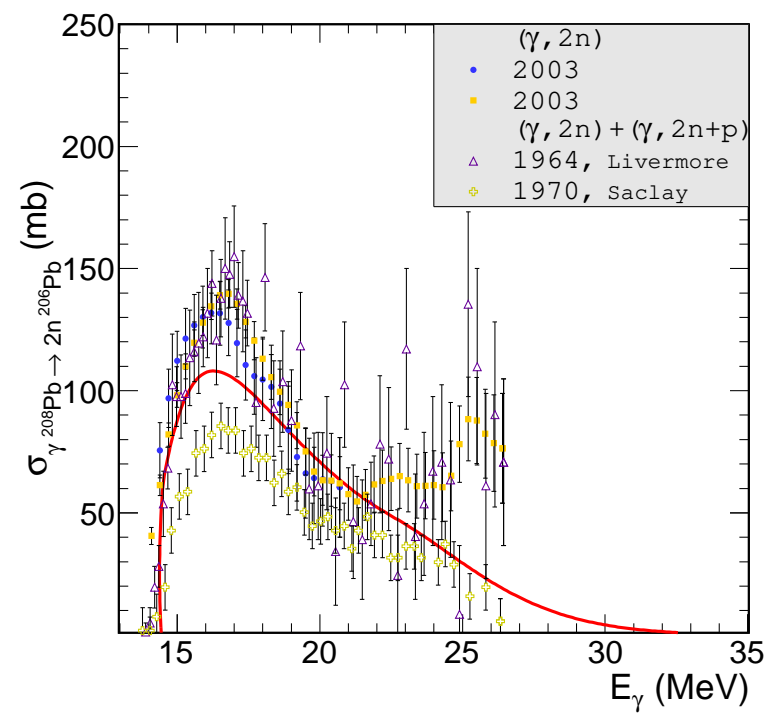

FIG. 8: Excitation energy for the $\gamma^{208} \mathrm{~Pb} \rightarrow 2 \mathrm{n}^{206} \mathrm{~Pb}$ reaction. Experimental data are from Refs. [23 25].

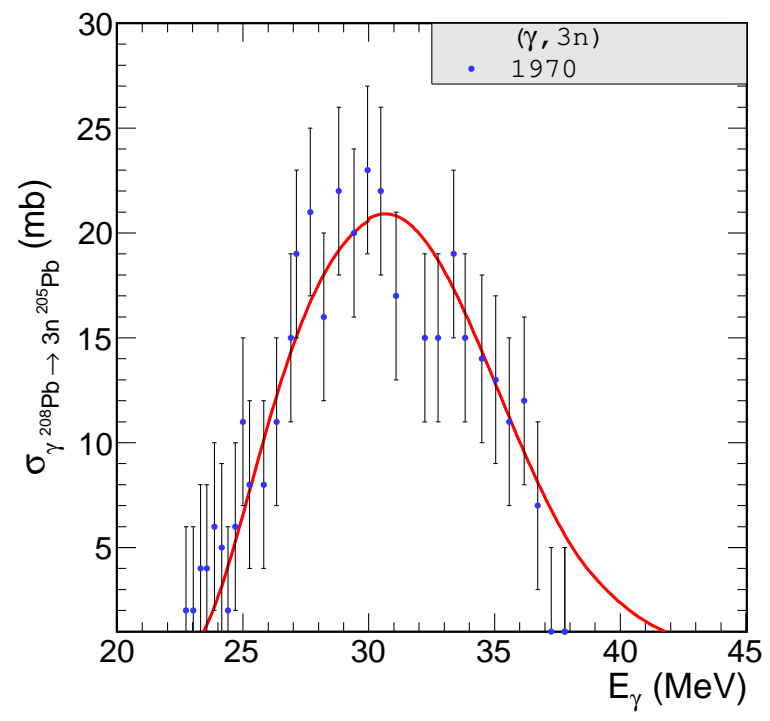

FIG. 9: Excitation function for the $\gamma^{208} \mathrm{~Pb} \rightarrow 3 \mathrm{n}^{205} \mathrm{~Pb}$ reaction. Experimental data are from Ref. [24]. 


\section{RESULTS FOR ELECTROMAGNETIC EXCITATIONS IN UPCS}

Now we shall present our results for electromagnetic excitation of nuclei in UPCs.

In Table II we have collected cross section in barns for single-nucleus single-photon excitation for different ranges of excitation energy and different collision energies represented by different $\gamma_{\mathrm{cm}}$ adequate for RHIC and LHC. In both cases the calculation was done for lead nuclei. Our results are compared with an earlier calculation by Vidović et al. [12]. Very good agreement can be observed. As already discussed, we are very interested also in cal-

TABLE II: Cross section in barns for single-nucleus, single-photon excitation for different ranges of excitation energy for ${ }^{208} \mathrm{~Pb}+{ }^{208} \mathrm{~Pb}$ collisions.

\begin{tabular}{|c|c|c|c|}
\hline & (6-40) $\mathrm{MeV}$ & $(40-2000)$ & -80) $\mathrm{GeV}$ \\
\hline \multicolumn{4}{|c|}{$\gamma_{\mathrm{cm}}=100$} \\
\hline M.Vidović et al. [12] & 77.6 & 25.7 & 5.6 \\
\hline Our results & 80.16 & 25.6 & 5.6 \\
\hline \multicolumn{4}{|c|}{$\gamma_{\mathrm{cm}}=3100$} \\
\hline M.Vidović et al. [12] & 133.6 & 53.7 & 18.7 \\
\hline Our results & 133.8 & 54.6 & 18.7 \\
\hline
\end{tabular}

culating associated neutron multiplicities. In Table III we have collected appropriate cross sections for one-photon single nucleus excitation in barns for different neutron multiplicities $\mathrm{k}=0,1,2,3$. We compare results with and without the exponential factor in Eq.(2.19). As can be seen from Table III the exponential factor plays here only a minor role in practical calculations. We have also collected experimental data of the ALICE collaboration [26]. We observe some disagreement especially for 3 neutrons. For the ratio $2 n / 1 n$ we obtain $22 \%$, in good agreement with the ALICE result of $22.5 \pm 0.5$ stat \pm 0.9 syst $\%$.

TABLE III: Cross section in barns for a given multiplicity of neutrons in single-nucleus, singlephoton excitation in ${ }^{208} \mathrm{~Pb}+{ }^{208} \mathrm{~Pb}$ collisions at $\sqrt{s_{N N}}=2.76 \mathrm{TeV}$.

\begin{tabular}{|c||r|r|r|}
\hline \multicolumn{4}{|c|}{ Single excitations [b] } \\
\hline \hline & Our results & $\exp (-\bar{n})$ & ALICE (Ref. [26]) \\
\hline 0 neutrons & 6.403 & without & \\
& 6.394 & with & \\
\hline 1 neutron & 84.301 & without & \\
& 82.888 & with & \\
\hline 2 neutrons & 18.608 & without & \\
& 18.532 & with & \\
\hline 3 neutrons & 2.858 & without & \\
& 2.856 & with & \\
\hline \hline sum & 112.170 & without & \\
& 110.670 & with & \\
\hline \hline total & & & \\
\hline
\end{tabular}


Two-photon exchanges may also lead to simultaneous excitation of both nuclei (see Fig. 3). In Table IV] we have collected topological cross sections with a given number of neutrons emitted from first $\left(k_{1}\right)$ and second $\left(k_{2}\right)$ nucleus. As previously we show results of the calculation with and without the extra exponential factor, which seems here more important than for single-nucleus single-photon excitations. This indicates an importance of smaller impact parameters in the two-photon processes. Our results could be compared to those in Ref. [6]. Compared to Ref. [6] our cross section for neutron multiplicities $k_{1}=1$ and $k_{2}=1$ are larger by about $25 \%$. Other numbers seem to be in much better agreement. The differences quantify the uncertainties of theoretical calculations.

TABLE IV: Cross section in barns for mutual excitation with a given number of neutrons emitted from both nuclei in ${ }^{208} \mathrm{~Pb}+{ }^{208} \mathrm{~Pb}$ collisions at $\sqrt{s_{N N}}=2.76 \mathrm{TeV}$.

\begin{tabular}{|c||c|c|c|c|c|}
\hline \multicolumn{7}{|c|}{ Mutual excitations [b] } \\
\hline & 0 neutrons & 1 neutron & 2 neutrons & 3 neutrons & $\exp (-\bar{n})$ \\
\hline \hline 0 neutrons & 0.00917 & 0.12093 & 0.02675 & 0.00414 & without \\
& 0.00883 & 0.09317 & 0.02483 & 0.00402 & with \\
\hline 1 neutron & 0.12093 & 1.59450 & 0.35275 & 0.05448 & without \\
& 0.09317 & 1.00124 & 0.26286 & 0.04238 & with \\
\hline 2 neutrons & 0.02675 & 0.35275 & 0.07803 & 0.01205 & without \\
& 0.02483 & 0.26286 & 0.06989 & 0.01130 & with \\
\hline 3 neutrons & 0.00413 & 0.05448 & 0.01205 & 0.00186 & without \\
& 0.00402 & 0.04238 & 0.01130 & 0.00183 & with \\
\hline \hline sum & 0.16098 & 2.12266 & 0.46958 & 0.07252 & without \\
& 0.19285 & 1.39965 & 0.36888 & 0.05953 & with \\
\hline \multicolumn{7}{|c||}{2.82574} & without \\
& \multicolumn{7}{|c|}{2.02091} & with \\
\hline \hline
\end{tabular}

In Table $\mathrm{V}]$ we have collected contributions to the double-photon excitation cross section when each of the two photons is in different energy intervals (the same integrals as defined previously in Table (II). The nine different regions in $\left(\mathrm{E}_{1} \times \mathrm{E}_{2}\right)$ space give comparable contribution to the total cross section for double-excitation of a single nucleus. It is particularly interesting how nuclei are excited in UPCs.

In Fig. 10 we show the total cross section for electromagnetic excitation as a function of $\sqrt{s_{N N}}$ as well as the partial cross sections into one and two-neutron final states. It should be noted that we concentrate only on the neutrons evaporated from the electromagnetically excited nuclei. We do not account for neutrons from other hadronic processes, like the intranuclear cascading (see for example [6, 27]). We also neglect the mutual excitation of nuclei by strong interactions.

\begin{tabular}{|c|}
\hline $\begin{array}{c}\text { Mutual excitations [b] } \\
\text { ALICE (Ref. [26]) }\end{array}$ \\
\hline 5.7 \\
\hline
\end{tabular}


TABLE V: Cross section in barns for double-photon excitation of one of nuclei in ${ }^{208} \mathrm{~Pb}+{ }^{208} \mathrm{~Pb}$ collisions at $\sqrt{s_{N N}}=2.76 \mathrm{TeV}$.

\begin{tabular}{|l||r|r||r|r|}
\hline & $E_{1}=(6-40) \mathrm{MeV}$ & $E_{1}=(40-2000) \mathrm{MeV}$ & $E_{1}=(2-80) \mathrm{GeV}$ & sum \\
\hline$E_{2}=(6-40) \mathrm{MeV}$ & 2.893 & 1.438 & 0.723 & 5.054 \\
$E_{2}=(40-2000) \mathrm{MeV}$ & 1.438 & 0.716 & 0.357 & 2.511 \\
$E_{2}=(2-80) \mathrm{GeV}$ & 0.723 & 0.357 & 0.178 & 1.258 \\
\hline \hline & 5.054 & 2.511 & 1.258 & 8.823 \\
\hline \hline
\end{tabular}

In Fig. 11 we show our result for ${ }^{208} \mathrm{~Pb}+{ }^{208} \mathrm{~Pb} \rightarrow{ }^{208} \mathrm{~Pb}^{*}+{ }^{208} \mathrm{~Pb}$ reaction at the LHC energy $\sqrt{s_{N N}}=2.76 \mathrm{TeV}$. For technical reasons different regions (low $(6-40 \mathrm{MeV}$ ), intermediate (40-2000 MeV), high $(>2 \mathrm{GeV})$ ) of excitation energy were calculated separately. The dashed line represents contribution of single-photon excitation (diagram in Fig. 1) and the dotted line double-photon excitation (diagram in Fig. 2). Even at the very high collision energy $\left(\sqrt{s_{N N}}=2.76 \mathrm{TeV}\right)$ the low-energy excitations are still essential. Please note, however, the logarithmic scale for the excitation energy axis, which emphasises the low-energy excitation. The double-photon excitation contribution is much smaller than the single-photon one. In addition, the highest peak appears at the excitation energy twice larger than for single-photon excitation, which corresponds to excitation of giant dipole resonance excited on top of an already excited one. Such processes were already discussed in the literature ([6] and the references therein).

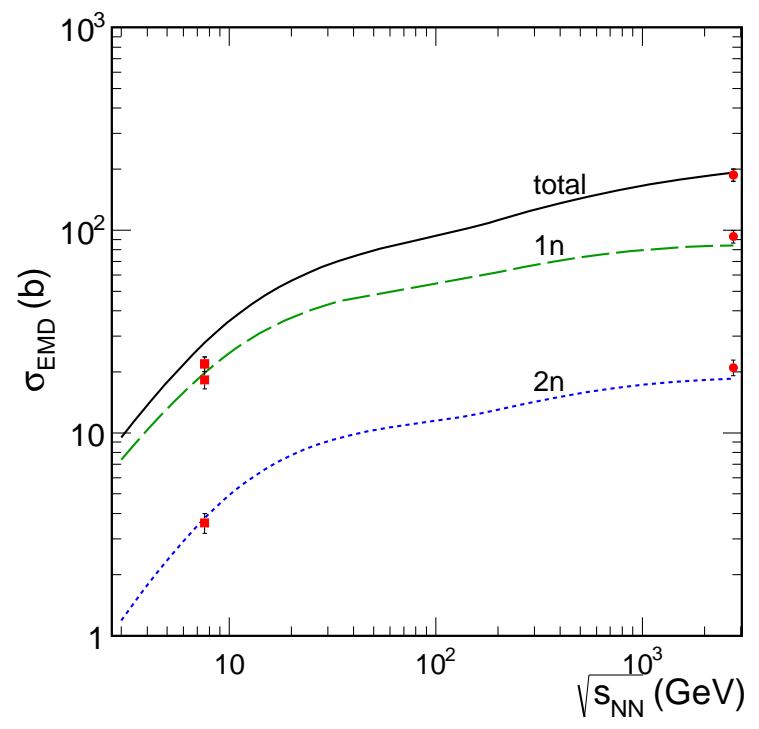

FIG. 10: Single EMD cross sections as the function of $\sqrt{s_{N N}}$. Data are from SPS [28] and LHC (ALICE) 26]. 


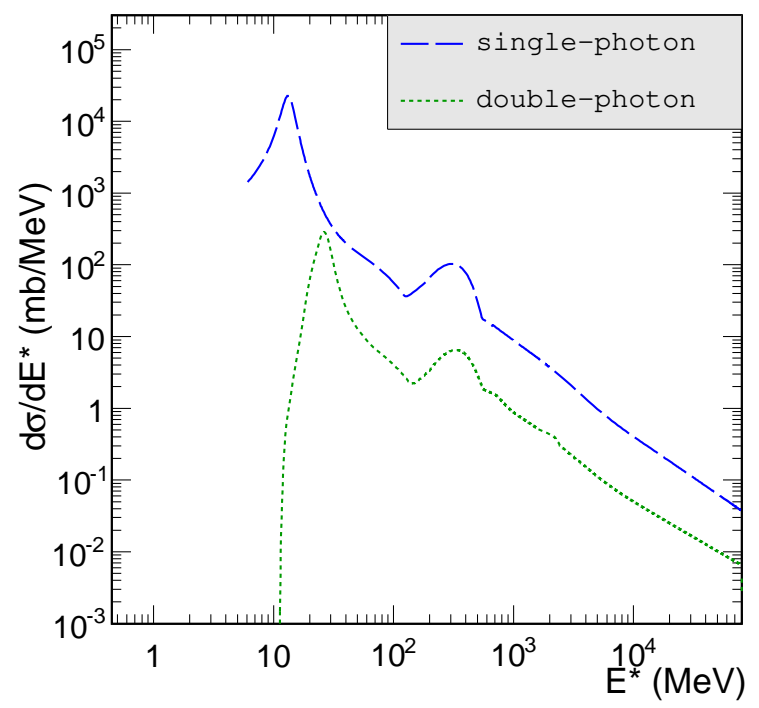

FIG. 11: Excitation function $\frac{d \sigma}{d E^{*}}$ for electromagnetic excitation of single nucleus in single-photon (dashed) and double-photon (dotted) exchange in UPCs at $\sqrt{s_{N N}}=2.76 \mathrm{TeV}$.

\section{CONCLUSIONS}

In this paper we have presented a new approach for calculating excitation of lead nuclei in photoabsorption reactions as well as in ultraperipheral ultrarelativistic heavy ion collisions. The photoabsorption cross section on lead nuclei is fitted using physics motivated multicomponent parametrization. The giant resonances, quasi-deuteron, excitation of nucleon resonances and break up of nucleon mechanism are included in the fit to the world data. Good quality fit is obtained.

The neutron emission from the excited nuclear system is calculated within the HauserFeshbach formalism. Within our approach we get a very good description of excitation functions for $\gamma+{ }^{208} \mathrm{~Pb}$ with a fixed number of neutrons. The excitation function is used next to calculate cross sections in peripheral UPCs. Both single and double-photon excitation processes are included and discussed. We have calculated corresponding excitation functions for both single (one-nucleus) and mutual (both nuclei) excitations.

We have obtained a good agreement of the calculated total cross section for electromagnetic excitation as well as cross section for one-neutron and two-neutron emission with recent experimental data of ALICE collaboration.

The formalism presented here may be easily applied to other exclusive ultrarelativistic heavy ion processes such as: $A A \rightarrow A A J / \Psi, A A \rightarrow A A \rho^{0}, A A \rightarrow A A e^{+} e^{-}, A A \rightarrow A A \mu^{+} \mu^{-}$. $A A \rightarrow A A \pi^{+} \pi^{-}, A A \rightarrow A A \pi^{+} \pi^{-} \pi^{+} \pi^{-}$. This will be discussed in our future analyses.

\section{Acknowledgments}

We would like to thank Igor Pshenichnov for the correspondence and Christoph Mayer and Joakim Nystrand for discussions. This work was partially supported by the Polish grant N N202 236640 and DEC-2011/01/B/ST2/04535 as well as by the Centre for Innovation and Transfer of Natural Sciences and Engineering Knowledge in Rzeszów. A part of the 
calculations within this analysis was carried out with the help of cloud computer system (Cracow Cloud One ${ }^{1}$ ) of the Institute of Nuclear Physics (PAN).

[1] V.M. Budnev, I.F. Ginzburg, G.V. Meledin and V.G. Serbo, Phys. Rep. 15 (1975) 4; C.A. Bertulani and G. Baur, Phys. Rep. 163 (1988) 29; G. Baur, K. Hencken, D. Trautmann, S. Sadovsky and Y. Kharlov, Phys. Rep. 364 (2002) 359; C.A. Bertulani, S.R. Klein and J. Nystrand, Ann. Rev. Nucl. Part. Sci. 55 (2005) 271; A.J. Baltz, G. Baur, D. d'Enterria et al., Phys. Rep. 458 (2008) 1.

[2] M. Kłusek, A. Szczurek and W. Schäfer, Phys. Lett. B674 (2009) 92; M. Kłusek-Gawenda and A. Szczurek, Phys. Rev. C82 (2010) 014904; M. Kłusek-Gawenda, A. Szczurek, M.V.T. Machado and V.G. Serbo, Phys. Rev. C83 (2011) 024903; S. Baranov, A. Cisek, M. KłusekGawenda, W. Schäfer and A. Szczurek, Eur. Phys. J. C73 (2013) 2335; M. Kłusek-Gawenda and A. Szczurek, Phys. Lett. B700 (2011) 322; M. Kłusek-Gawenda and A. Szczurek, Phys. Rev. C87 (2013) 054908; M. Kłusek-Gawenda and A. Szczurek, arXiv: 1309.2463 [nucl-th].

[3] C. Adler, A. Denisov, E. Garcia, M. Murray, H. Stroebele and S. White, Nucl. Instrum. Meth. A470 (2001) 488.

[4] K. Aamodt et al. (ALICE Collaboration), JINST 3 (2008) S08002.

[5] W.J. Llope and P. Braun Munzinger, Phys. Rev. C41 (1990) 2644.

[6] I.A. Pshenichnov, Phys. Part. Nucl. 42 (2011) 215.

[7] G. Baur, K. Hencken, A. Aste, D. Trautmann and S. R. Klein, Nucl. Phys. A 729 (2003) 787.

[8] V.A. Plujko, R. Capote and O.M. Gorbachenko, At. Data and Nucl. Data Tables 97 (2011) 567.

[9] J. Speth and A. van der Woude, Rep. Progr. Phys. 44 (1988) 719.

[10] V.V. Varlamov, M.E. Stepanov and V.V. Chesnokov, Izv. Ross. Akad. Nauk, Ser. Fiz. 67 (2003) 656.

[11] M.B. Chadwick, P. Oblozinsky, P.E. Hodgson and G. Reffo, Phys. Rev. C44 (1991) 814.

[12] M. Vidović, M. Greiner and G. Soff, Phys. Rev. C48 (1993) 2011.

[13] J. Ahrens, Nucl. Phys. A446 (1985) 229c.

[14] R.J. Charity, Phys. Rev. C82 (2010) 014610.

[15] W. Hauser, H. Feshbach, Phys. Rev. 87 (1952) 366.

[16] P. Möller, J.R. Nix, W.D. Myers, and W.J. Swiatecki, At. Data Nucl. Data Tables 59 (1995) 185.

[17] L. M. Young, Phd dissertation (1972), University of Illinois at Urbana-Champaign ("Photoneutron Cross Sections and Spectra from Monoenergetic Photons on Y, Pr, Pb, and Bi in the Giant Resonance").

[18] R. Van de Vyver, J. Devos, H. Ferdinande, R. Carchon and E. Van Camp, Z.Phys. A284 (1978) 91.

[19] S.N. Belyaev, A.B. Kozin, A.A. Nechkin, V.A. Semenov and S.F. Semenko, Yad. Fiz. 42 (1985) 1050.

[20] S.N. Belyaev, O.V. Vasilev, A.B. Kozin, A.A. Nechkin and V.A. Semenov, Program and Theses, Proc.35th Ann. Conf. Nucl. Spectrosc. Struct. At. Nuclei, Leningrad, (1985) p.351.

\footnotetext{
${ }^{1}$ cc1.ifj.edu.pl
} 
[21] S.N. Belyaev, A.A. Nechkin and V.A. Semenov, Izv. Rossiiskoi Akademii Nauk, Ser.Fiz. 55 (1991) 953.

[22] V.V. Varlamov, N.G. Efimkin, B.S. Ishkhanov and V.V. Sapunenko, Nuclear Constants 1 (1993) 52 .

[23] R.R. Harvey, J.T. Caldwell, R.L. Bramblett and S.C. Fultz, Phys. Rev. B136 (1964) 126.

[24] A. Veyssiere, H. Beil, R. Bergere, P. Carlos and A. Lepretre, Nucl. Phys. A159 (1970) 561.

[25] V.V. Varlamov, N.N. Peskov, D.S. Rudenko and M.E. Stepanov, YK (1-2) (2003) 48.

[26] B. Abelev et al. (ALICE Collaboration), Phys. Rev. Lett. 109 (2012) 252302.

[27] I. A. Pshenichnov, J. P. Bondorf, I. N. Mishustin, A. Ventura and S. Masetti, Phys. Rev. C 64 (2001) 024903.

[28] M. B. Golubeva, F. F. Guber, T. L. Karavicheva, E. V. Karpechev, A. B. Kurepin, A. I. Maevskaya, I. A. Pshenichnov and A. I. Reshetin et al., Phys. Rev. C 71 (2005) 024905. 